\title{
150-Water Dynamic Positron Emission Tomography in Patients with Non-Small Cell Lung Cancer: Early Decrease in Blood Flow After Anti-Angiogenic Treatment is Correlated with a Shorter Time to Tumour Progression
}

\section{Daisuke Katayama}

Osaka University Graduate of School of Medicine

Masahiro Yanagawa ( $\nabla$ m-yanagawa@radiol.med.osaka-u.ac.jp )

https://orcid.org/0000-0002-0911-6769

\section{Keiko Matsunaga}

Osaka University Graduate School of Medicine

Hiroshi Watabe

Tohoku University

\section{Tadashi Watabe}

Osaka University Graduate School of Medicine

\section{Hiroki Kato}

Osaka University Graduate School of Medicine

\section{Takashi Kijima}

Hyogo College of Medicine

Yoshito Takeda

Osaka University Graduate School of Medicine

Atsushi Kumanogoh

Osaka University Graduate School of Medicine

Eku Shimosegawa

Osaka University Graduate School of Medicine

Jun Hatazawa

Osaka University

Noriyuki Tomiyama

Osaka University Graduate School of Medicine

Original research

Keywords: bevacizumab, blood flow, non-small cell lung cancer, NSCLC, progression-free survival 
Posted Date: July 17th, 2020

DOl: https://doi.org/10.21203/rs.3.rs-41557/v1

License: (c) (i) This work is licensed under a Creative Commons Attribution 4.0 International License. Read Full License 


\section{Abstract}

Purpose: To evaluate tumour blood flow in patients with non-small cell lung cancer (NSCLC) who underwent chemotherapy with bevacizumab before and after administration of chemotherapy using ${ }^{15} \mathrm{O}$ water positron emission tomography (PET), and to investigate the effects of bevacizumab on tumour blood flow changes and progression-free survival (PFS).

Methods: Twelve patients with NSCLC were enrolled. Six patients had chemotherapy with bevacizumab, and the other six had chemotherapy without bevacizumab. ${ }^{15} \mathrm{O}$-water dynamic PET scans were performed within 1 week before the start of chemotherapy and within 1 week after the first day of chemotherapy. Tumour blood flow was analysed quantitatively using a single one-tissue compartment model with the correction of pulmonary circulation blood volume and arterial blood volume via an imagederived input function.

Results: In the bevacizumab group mean tumour blood flow had reduced statistically significantly postchemotherapy (pre-chemotherapy $0.27 \pm 0.14 \mathrm{~mL} / \mathrm{cm}^{3} / \mathrm{min}$, post-chemotherapy $0.18 \pm 0.12$ $\mathrm{mL} / \mathrm{cm}^{3} / \mathrm{min}$ ). In the no bevacizumab group there was no significant difference between mean tumour perfusion pre-chemotherapy $\left(0.42 \pm 0.42 \mathrm{~mL} / \mathrm{cm}^{3} / \mathrm{min}\right)$ and post-chemotherapy $(0.40 \pm 0.27$ $\mathrm{mL} / \mathrm{cm}^{3} / \mathrm{min}$ ). In the bevacizumab group there was a positive correlation between the blood flow ratio (tumour blood flow post-chemotherapy/tumour blood flow pre-chemotherapy) and PFS (correlation coefficient 0.94).

Conclusion: Mean tumour blood flow decreases within 1-2 days after bevacizumab administration. Greater reductions in blood flow were associated with shorter PFS.

\section{Introduction}

Vascular endothelial growth factor (VEGF) is a major contributor to angiogenesis, which plays important roles in local tumour progression and metastatic growth. Overexpression of VEGF has been observed in a variety of cancers including non-small cell lung cancer (NSCLC). Bevacizumab is a monoclonal anti-VEGF antibody that inhibits angiogenesis by preventing circulating VEGF from binding to its receptors (Ferrara et al., 2004). The addition of bevacizumab to cytotoxic chemotherapy improves overall survival and progression-free survival (PFS), and is an accepted component of care for NSCLC [1]. Notably however, the mechanism by which the combination of bevacizumab and cytotoxic chemotherapy improves survival in cancer patients remains unclear.

Positron emission tomography (PET) with ${ }^{15} \mathrm{O}$-labelled water is an established method for measuring tissue blood flow quantitatively. ${ }^{15} \mathrm{O}$-water is an ideal tracer for quantifying blood flow because it is distributed to tissue freely and cannot be metabolized. Compared to other PET tracers such as ${ }^{82} \mathrm{Rb}$, the correlation between ${ }^{15} \mathrm{O}$-water-derived values and actual blood flow is very high [2]. Previously ${ }^{15} \mathrm{O}$-water PET was an invasive examination because it required continuous arterial blood sampling during the scan 
to obtain the input function. Now the image-derived input function is commonly used however, which is a noninvasive and accurate alternative to arterial sampling [3]. PET using ${ }^{15} \mathrm{O}$-water is regarded as the gold standard for brain perfusion and it is now used in other areas such as myocardial perfusion and tumour perfusion $[4,5]$.

Imaging is commonly and widely used as a non-invasive procedure to evaluate treatment responses. The Response Evaluation Criteria in Solid Tumours (RECIST) are widely used to evaluate treatment responses, but in many cases the criteria are not appropriate for predicting the outcomes of novel molecularly targeted cancer treatments because they are based on the reduction rate of morphological size [6]. PET facilitates the visualization of metabolic activity in tumours, resulting in more informative evaluations of treatment responses after chemotherapy. ${ }^{15} \mathrm{O}$-water PET enables the measurement of tumour perfusion defined as blood flow $(\mathrm{mL} / \mathrm{min})$ per $\mathrm{cm}^{3}$. We hypothesized that when assessing treatment responses to bevacizumab it may be possible to determine therapeutic effects more accurately by evaluating changes in tumour blood flow than by evaluating changes in tumour size identified via computed tomography (CT).

The purpose of the current prospective study was to evaluate tumour blood flow in patients with NSCLC who underwent chemotherapy with or without bevacizumab before and after that chemotherapy using ${ }^{15} \mathrm{O}$-water PET, and to investigate the effects of bevacizumab on tumour blood flow and PFS.

\section{Materials And Methods}

\section{Patients}

Between April 2012 and July 2015 patients with stage IV NSCLC who were scheduled to undergo chemotherapy at the Osaka University Hospital in Osaka, Japan were recruited by respiratory physicians before the start of treatment. Of the 76 patients encountered during the study period, 13 agreed to participate in the study (Fig. 1). One patient withdrew from participation after providing consent. The final sample of subjects included 8 men and 4 women, with a mean age of 60 years (range 42-73 years). Ten patients had adenocarcinoma, 1 had pleomorphic carcinoma, and 1 had poorly differentiated carcinoma. The patient characteristics are summarized in Table 1. ${ }^{15} \mathrm{O}$-water PET is not covered by insurance in Japan. Approval from the internal Ethics Review Board was obtained before the initiation of the study. Informed consent was obtained from each patient. 
Table 1

Patient characteristics
Patient no.
Sex
Age Histological type
Chemotherapy regimen

Chemotherapy with bevacizumab

\begin{tabular}{|lllll|}
\hline 1 & M & 62 & adenocarcinoma & CBDCA + PTX + BEV \\
\hline 2 & F & 67 & adenocarcinoma & CBDCA + PTX + BEV \\
\hline 3 & M & 62 & adenocarcinoma & CBDCA + PTX + BEV \\
\hline 4 & M & 65 & adenocarcinoma & CBDCA + PTX + BEV \\
\hline 5 & F & 42 & adenocarcinoma & CBDCA + PEM + BEV \\
\hline 6 & M & 73 & adenocarcinoma & CBDCA + PEM + BEV \\
\hline Chemotherapy without bevacizumab & CBDCA + PEM \\
\hline 7 & M & 46 & adenocarcinoma & CBDCA + PEM \\
\hline 8 & M & 61 & adenocarcinoma & CBDCA + PTX \\
\hline 9 & M & 64 & poorly differentiated carcinoma & CBDCA + PTX \\
\hline 10 & F & 42 & pleomorphic carcinoma & CDDP + PEM \\
\hline 11 & M & 68 & adenocarcinoma & CBDCA + PEM \\
\hline 12 & M & 71 & adenocarcinoma & \\
\hline $\begin{array}{l}\text { M, male; F, female; CBDCA, carboplatin; PTX, paclitaxel; BEV, bevacizumab; PEM, pemetrexed; CDDP, } \\
\text { cisplatin }\end{array}$
\end{tabular}

\section{Chemotherapy}

Initial chemotherapy was administered to all the patients in the study in hospital. The chemotherapy regimens-including the use of bevacizumab-depended on the respiratory physicians treating each individual patient. Six patients (4 men and 2 women, mean age $62 \pm 10$ years) underwent chemotherapy that included bevacizumab, and the other 6 ( 4 men and 2 women, mean age $59 \pm 11$ years) had chemotherapy without bevacizumab. All patients in the bevacizumab group were diagnosed with adenocarcinoma histologically. Four of them were administered bevacizumab with carboplatin and paclitaxel, and 2 of them were administered bevacizumab with carboplatin and pemetrexed. The histological types in the no bevacizumab group included 4 adenocarcinomas, 1 pleomorphic carcinoma and 1 poorly differentiated carcinoma. The chemotherapy regimens are shown in Table 1 . The bevacizumab dose was $15 \mathrm{mg} / \mathrm{kg}$ of body weight. The doses of the other anticancer agents were carboplatin AUC 6, paclitaxel $200 \mathrm{mg} / \mathrm{m}^{2}$, pemetrexed $500 \mathrm{mg} / \mathrm{m}^{2}$ and cisplatin $80 \mathrm{mg} / \mathrm{m}^{2}$. In most regimens all drugs were administered on day 1 , and in all regimens the next administration was 3 weeks thereafter. 


\section{PET/CT imaging protocol}

${ }^{15} \mathrm{O}$-water dynamic PET scans were obtained as a prospective study designed to evaluate tumour blood flow before and after bevacizumab administration. Baseline PET was performed within 1 week before the start of chemotherapy (mean 3.8 days, range 1-6 days). Post-chemotherapy PET was performed within 1 week after the first day of administration (mean 1.7 days, range 1-6 days). PET imaging was acquired on a SET-3000 GCT/X scanner (Shimadzu Corp., Kyoto, Japan). This scanner has an axial field of view of $26 \mathrm{~cm}$, divided into 99 contiguous planes. The intrinsic spatial resolution is $3.5 \mathrm{~mm}$ full width at half maximum (FWHM) in-plane, and $4.2 \mathrm{~mm}$ FWHM axially.

Patients were positioned supine in the scanner bed with both the tumour and the aortic arch or heart in the centre of the axial field of view. For attenuation correction a 5-min transmission scan was performed using a ${ }^{137} \mathrm{Cs}$ point source. After that transmission scan a 10-min list mode scan was started simultaneously with an intravenous injection of $185 \mathrm{MBq}$ of ${ }^{15} \mathrm{O}$-water (total amount $18.5 \mathrm{~mL}$, injection speed $0.5 \mathrm{~mL} / \mathrm{s}$ ). The emission scan was reconstructed into 22 frames ( 1 frame $\times 10 \mathrm{~s}, 8$ frames $\times 5 \mathrm{~s}, 4$ frames $\times 10 \mathrm{~s}, 2$ frames $\times 15 \mathrm{~s}, 3$ frames $\times 20 \mathrm{~s}, 2$ frames $\times 30 \mathrm{~s}$ and 6 frames $\times 60 \mathrm{~s}$ ) using the twodimensional dynamic row-action maximumlikelihood algorithm after three-dimensional Gaussian smoothing with a 6-mm FWHM. The voxel size was $4.7 \times 4.7 \times 2.6 \mathrm{~mm}$.

After the emission scan, reference CT was performed. The CT acquisition parameters were breath hold at shallow inspiration, from the apex of the lung to the base of the lung, no intravenous media, $120 \mathrm{kVp}$ and 50 effective mAs, 52 slices, and 5.0-mm slice thickness. Clinically baseline CT scanning was performed up to 1 month before the start of chemotherapy. Follow-up CT scans were performed after 2 courses of chemotherapy and every 2-4 months thereafter. Brain magnetic resonance imaging and bone scintigraphy were performed as needed. PFS was defined as the time from the start of treatment to the appearance of local recurrence or new metastatic lesions.

\section{Quantitative analysis for tumour blood flow}

Tumour perfusion was calculated via the following equation (Eq. 1), using a single one-tissue compartment model with the correction of pulmonary circulation blood volume and arterial blood volume [3].

$C_{P E T}(\mathrm{t})=\left(1-V_{A}^{\square} V_{V}\right) \otimes F \square C_{A}(\mathrm{t}) \otimes e^{-(F / V T) t}+V_{A} C_{A}(\mathrm{t})+V_{V} C_{V}(\mathrm{t})$ Eq. 1

Where:

$C_{P E T}(\mathrm{t})=$ tracer concentration in tissue $\left(\mathrm{Bq} / \mathrm{cm}^{3}\right), C_{A}(\mathrm{t})=$ tracer concentration in artery $\left(\mathrm{Bq} / \mathrm{cm}^{3}\right), C_{V}(\mathrm{t})=$ tracer concentration in the right ventricular cavity $\left(\mathrm{Bq} / \mathrm{cm}^{3}, F=\right.$ blood flow $\left(\mathrm{mL} / \mathrm{cm}^{3} / \mathrm{min}\right), V_{T}=$ partition coefficient of water, $V_{A}=$ arterial blood volume, and $V_{V}=$ pulmonary circulation blood volume. 
The image-derived input function was used. Volumes of interest (VOIs) with a diameter of $1 \mathrm{~cm}$ were drawn over the ascending aorta in approximately 10 consecutive image planes of the frame in which the first pass of the bolus was best visualized. Projection of the resulting VOI onto all image frames yielded the arterial time-activity curve or image-derived input function $C_{A}(\mathrm{t})$. Applying a similar approach to the right ventricular cavity provided a time-activity curve for the pulmonary circulation $\mathrm{C}_{\mathrm{V}}(\mathrm{t})$. Parametric images of perfusion were generated using the basis function method [7]. In the present study 300 logarithmically spaced precomputed basis functions with F/VT values ranging from 0.0 to $0.4 \mathrm{~min}^{-1}$ were used. Tumour VOIs were defined on the reference $C T$ and projected onto the parametric perfusion images using PMOD 3.6 (PMOD Technologies, LLC, Zürich, Switzerland). The averaged tumour perfusion over the VOI in the parametric perfusion images was used for statistical analyses.

\section{Statistical analysis}

Tumour blood flow before and after chemotherapy were compared in the bevacizumab group and the no bevacizumab group. In the bevacizumab group tumour blood changes and PFS were examined. All statistical analyses were performed using commercially available software (MedCalc version 19.2.1, Frank Schoonjans, Mariakerke, Belgium). Differences in mean tumour blood flow before and after chemotherapy in each group were analysed using the Wilcoxon test. In the bevacizumab group regression analysis was performed on the blood flow ratio before and after chemotherapy and PFS. $p<0.05$ was considered statistically significant.

\section{Results}

In the bevacizumab group mean tumour perfusion was statistically significantly reduced after chemotherapy (pre-chemotherapy $0.27 \pm 0.14 \mathrm{~mL} / \mathrm{cm}^{3} / \mathrm{min}$, post-chemotherapy $0.18 \pm$ $\left.0.12 \mathrm{~mL} / \mathrm{cm}^{3} / \mathrm{min}\right)$. In the no bevacizumab group there was no significant difference between mean tumour perfusion pre-chemotherapy $\left(0.42 \pm 0.42 \mathrm{~mL} / \mathrm{cm}^{3} / \mathrm{min}\right)$ and post-chemotherapy $(0.40 \pm$ $0.27 \mathrm{~mL} / \mathrm{cm}^{3} / \mathrm{min}$ ) (Fig. 2). Table 2 shows the tumour blood flow measurements in each patient. 
Table 2

Quantitative tumour blood flow values in each patient. $F_{\text {after }} / F_{\text {baseline }}$ is the ratio of tumor blood flow after treatment to tumor blood flow at baseline.

\begin{tabular}{|llll|}
\hline Patient no. & F baseline & F after treatment & $F_{\text {after }} / F_{\text {baseline }}$ \\
\hline Chemotherapy with bevacizumab & & \\
\hline 1 & 0.371 & 0.334 & 0.899 \\
\hline 2 & 0.256 & 0.059 & 0.231 \\
\hline 3 & 0.221 & 0.185 & 0.838 \\
\hline 4 & 0.301 & 0.171 & 0.567 \\
\hline 5 & 0.452 & 0.284 & 0.628 \\
\hline 6 & 0.047 & 0.039 & 0.824 \\
\hline Chemotherapy without bevacizumab & & \\
\hline 7 & 0.351 & 0.335 & 0.956 \\
\hline 8 & 1.225 & 0.838 & 0.684 \\
\hline 9 & 0.100 & 0.130 & 1.292 \\
\hline 10 & 0.449 & 0.514 & 1.145 \\
\hline 11 & 0.063 & 0.104 & 1.648 \\
\hline 12 & 0.358 & 0.474 & 1.322 \\
\hline F, tumour blood flow (mL/cm ${ }^{3} /$ min $^{2}$ & & \\
\hline
\end{tabular}

PFS was not significantly correlated with tumour blood flow before or after chemotherapy in either group. In the bevacizumab group the rate of decline in tumour blood flow varied markedly in different patients. There was a positive correlation between the blood flow ratio (post-chemotherapy tumour blood flow/prechemotherapy tumour blood flow) and PFS (correlation coefficient 0.94 ), yielding a regression equation of $y=0.2729+0.001616 x(p=0.005)$ (Fig. 3). A smaller blood flow ratio after chemotherapy was associated with a shorter time to tumour recurrence or regrowth (Figs. 4 and 5). In the no bevacizumab group there was no significant correlation between the blood flow ratio and PFS (correlation coefficient $0.74, p=0.091)$.

\section{Discussion}

In the present study bevacizumab was associated with significantly reduced tumour blood flow 1-2 days after chemotherapy. Notably however, follow-up investigation revealed that this effect was associated with rapid tumour regrowth. Patients with only a slight change in blood flow tended to exhibit longer PFS. 
${ }^{15} \mathrm{O}$-water PET was an optimal and non-invasive method for assessing treatment responses to chemotherapy regimens including bevacizumab in patients with NSCLC. The vascular normalization theory proposed by Jain [8] may explain this phenomenon. Unlike physiological angiogenesis processes such as wound healing, tumour angiogenesis continues abnormally while the tumour is growing because the tumour requires a vascular supply to provide essential nutrients and oxygen. Tumour vessels are often tortuous, disorganized and highly permeable, resulting in high interstitial pressure and reduced blood perfusion and oxygenation. Tumour cells can adapt to insufficient blood supply and hypoxia, but drug delivery is inhibited and its efficacy is reduced. Excessive antiangiogenic therapy may lead to reduced tumour blood flow and result in hypoxia and acidosis, which promote tumour progression [9]. Moderate anti-VEGF therapy may lead to 'vascular normalization', which is characterized by attenuation of hyperpermeability, increased vascular pericyte coverage, a more normal basement membrane, and a resultant reduction in tumour hypoxia and interstitial fluid pressure. As a result drug delivery of cytotoxic anticancer agents is improved, and consequently chemotherapy in combination with anti-VEGF drugs improves survival.

In the current study a decline in tumour blood flow after bevacizumab administration was observed in all patients, but patients with greater reductions in tumour blood flow exhibited tumour regrowth within shorter periods. It may be that greater reduction of tumour perfusion reflects greater pruning of vessels, which leads to hypoxia and acidosis in the tumour. Heist et al. [10] reported that reduced blood perfusion after bevacizumab administration as determined via CT was associated with shorter overall survival in NSCLC patients, which is consistent with the results of the present study. They assessed tumour perfusion before bevacizumab administration and 14 days thereafter. In the current study the assessment of tumour perfusion was performed in 1-2 days after bevacizumab administration. The present study suggests that it may be possible to predict the effects of chemotherapy just days after the administration of bevacizumab using ${ }^{15} \mathrm{O}$-water PET. If bevacizumab is found to be insufficiently effective at an early stage, switching to another anticancer drug could be considered earlier. In addition, ${ }^{15} \mathrm{O}$ water PET can be performed at a lower dose than perfusion CT; $0.37 \mathrm{mSv}$ vs. 3.5-6.5 mSv [11].

Tumour blood flow is still not well understood, so it is necessary to clarify how tumour blood flow before treatment and changes in tumour blood flow after treatment affect the efficacy of anticancer therapy, and prognoses. Accordingly, a larger number of cases needs to be examined. Tumour blood flow is associated with tumour hypoxia, which is associated with resistance to chemotherapy. An explanation for why the extent of the reduction in tumour blood flow is associated with the response to chemotherapy may be associated with the altered hypoxic region of the tumour. Further insights may be obtained by combining hypoxic imaging with a radiolabelled tracer such as ${ }^{18} \mathrm{~F}$-fluoromisonidazol [12].

The current study had several limitations. One is the small number of participants, which resulted in low statistical power. It was also conducted at a single facility, which may have resulted in selection bias. Each patient underwent different chemotherapy regimens, which may have influenced blood flow and prognoses. There were also some technical limitations. The parametric images obtained are prone to noise, which slightly reduces the reliability of the quantification. Each VOI was manually placed over the 
pulmonary nodules, making it as large as possible in an effort to minimize the effects of inhomogeneity. This may have resulted in some variability due to manual measurement.

In conclusion, in the current study mean tumour blood flow diminished within 1-2 days after bevacizumab administration in NSCLC patients, and greater reductions in blood flow were associated with shorter PFS.

\section{Declarations}

\section{Funding}

This work was supported by a Grant-in-Aid for Scientific Research (A) (grant number 17H01575) and The Osaka Medical Research and Foundation for Intractable Diseases. Jun Hatazawa received a Grant-in-Aid for Scientific Research (A). Daisuke Katayama received a grant from The Osaka Medical Research and Foundation for Intractable Diseases. The other authors state that they have not received any funding for this work.

\section{Ethics approval and consent to participate}

All procedures performed were approved by the ethical committee of Osaka University Hospital (No.11109). Informed consent was obtained from all individual participants included in the study.

\section{References}

1. Sandler A, Gray R, Perry MC, et al. Paclitaxel-carboplatin alone or with bevacizumab for non-smallcell lung cancer. N Engl J Med. 2006;355:2542-50.

2. Maddahi J, Packard RR. Cardiac PET perfusion tracers: current status and future directions. Semin Nucl Med. 2014;44:333-43.

3. van der Veldt AA, Hendrikse $\mathrm{NH}$, Harms $\mathrm{HJ}$, et al. Quantitative parametric perfusion images using 150-labeled water and a clinical PET/CT scanner: test-retest variability in lung cancer. J Nucl Med. 2010;51:1684-90.

4. Harms HJ, de Haan S, Knaapen P, Allaart CP, Lammertsma AA, Lubberink M. Parametric images of myocardial viability using a single ${ }^{15} \mathrm{O}-\mathrm{H}_{2} \mathrm{O}$ PET/CT scan. J Nucl Med. 2011;52:745-9.

5. Tolbod LP, Nielsen MM, Pedersen BG, et al. Non-invasive quantification of tumor blood flow in prostate cancer using ${ }^{15} \mathrm{O}_{2} \mathrm{H}_{2} \mathrm{O}$ PET/CT. Am J Nucl Med Mol Imaging. 2018;8:292-302.

6. Nishino M, Jagannathan JP, Krajewski KM, et al. Personalized tumor response assessment in the era of molecular medicine: cancer-specific and therapy-specific response criteria to complement pitfalls of RECIST. AJR Am J Roentgenol. 2012;198:737-45. 
7. Watabe $\mathrm{H}$, Jino $\mathrm{H}$, Kawachi $\mathrm{N}$, et al. Parametric imaging of myocardial blood flow with ${ }^{15} \mathrm{O}$-water and PET using the basis function method. J Nucl Med. 2005;46:1219-24.

8. Jain RK. Normalization of tumor vasculature: an emerging concept in antiangiogenic therapy. Science. 2005;307:58-62.

9. Jain RK. Antiangiogenesis strategies revisited: from starving tumors to alleviating hypoxia. Cancer Cell. 2014;26:605-22.

10. Heist RS, Duda DG, Sahani DV, et al. Improved tumor vascularization after anti-VEGF therapy with carboplatin and nab-paclitaxel associates with survival in lung cancer. Proc Natl Acad Sci USA. 2015;112:1547-52.

11. Ketelsen D, Horger M, Buchgeister M, et al. Estimation of radiation exposure of 128-slice 4D-perfusion CT for the assessment of tumor vascularity. Korean J Radiol. 2010;11:547-52.

12. Xu Z, Li XF, Zou H, Sun X, Shen B. ${ }^{18} \mathrm{~F}$-fluoromisonidazole in tumor hypoxia imaging. Oncotarget. 2017;8:94969-79.

\section{Figures}

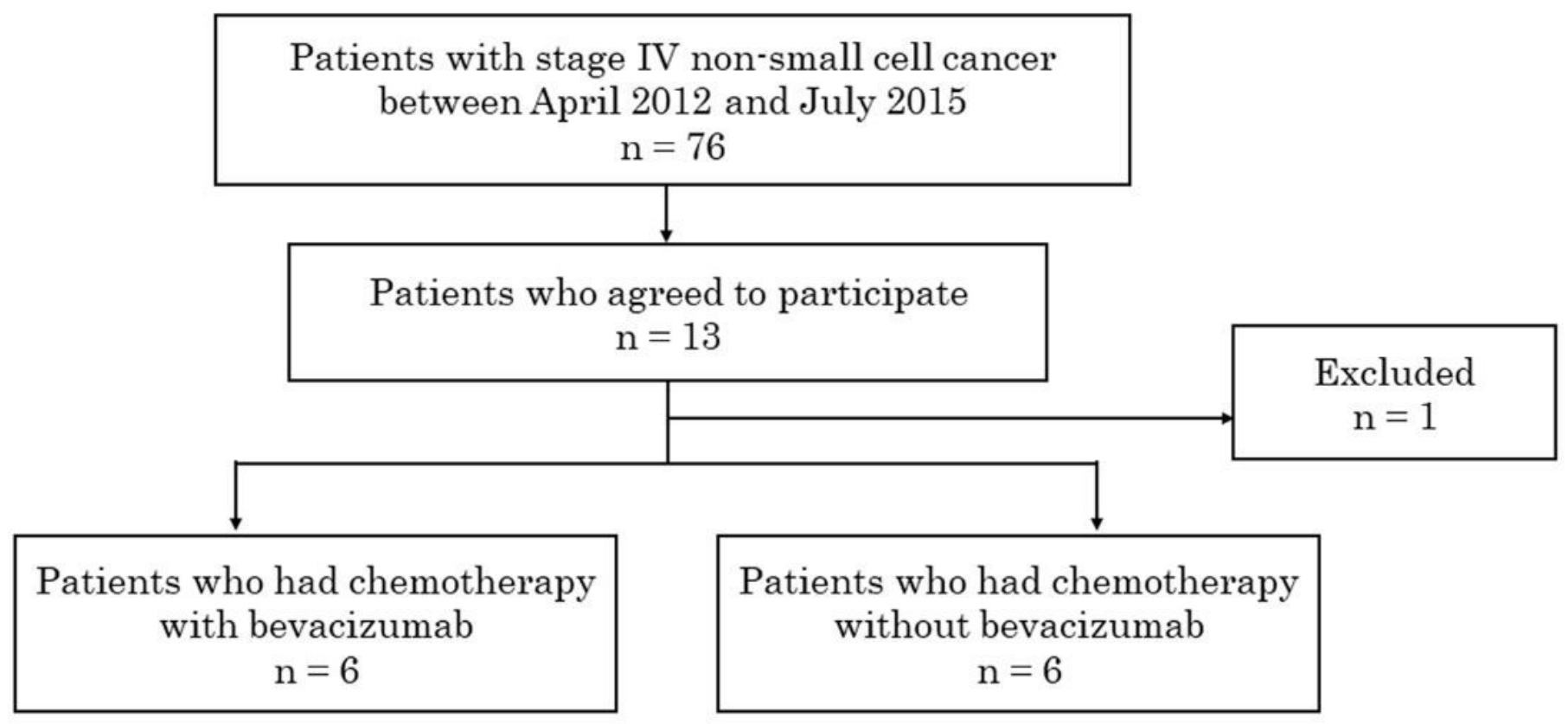

\section{Figure 1}

Study flowchart 
(A) Patient with Bevacizumab

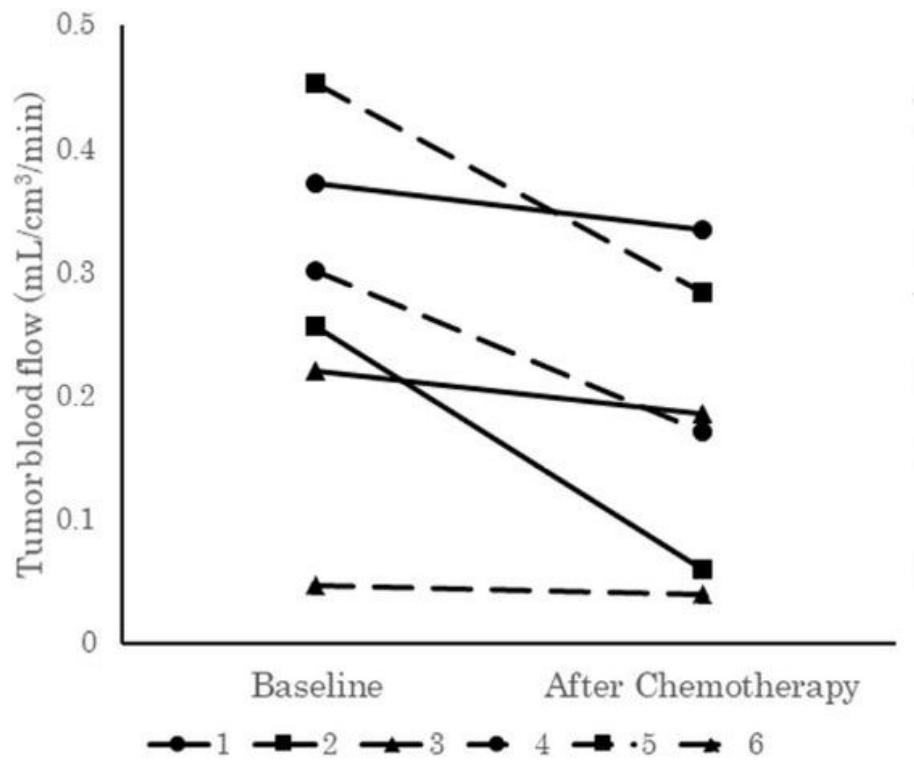

(B) Patient without Bevacizumab

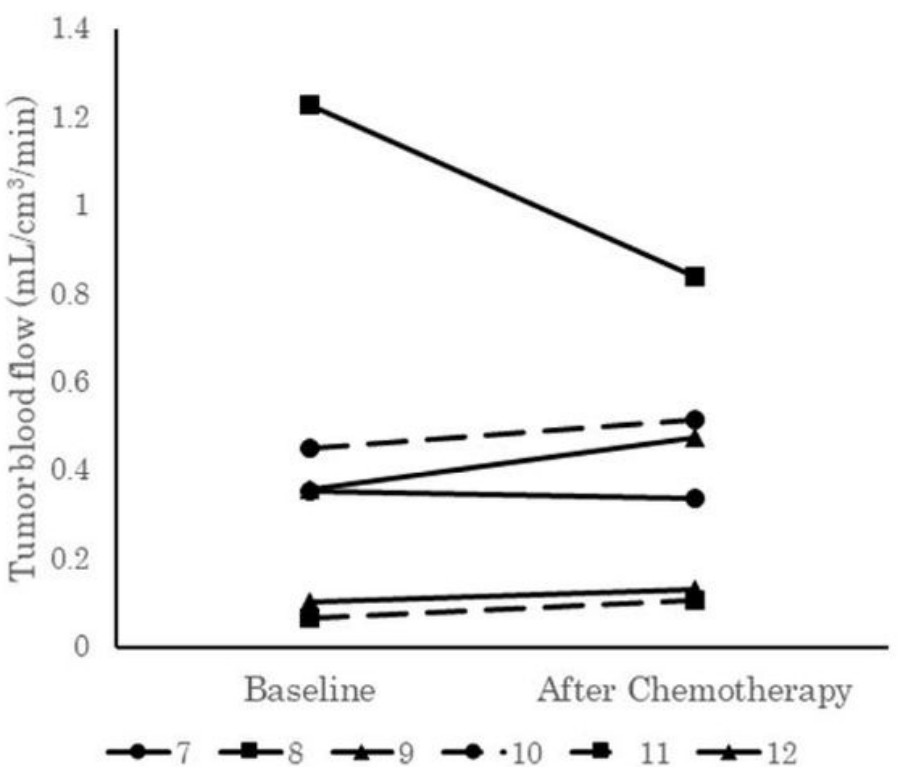

Figure 2

Tumor blood flow before and after chemotherapy in each patient (A) Patients who underwent chemotherapy with bevacizumab. There was a statistically significant decrease in blood flow. (B) Patients who underwent chemotherapy without bevacizumab. There was no statistically significant change in blood flow. 


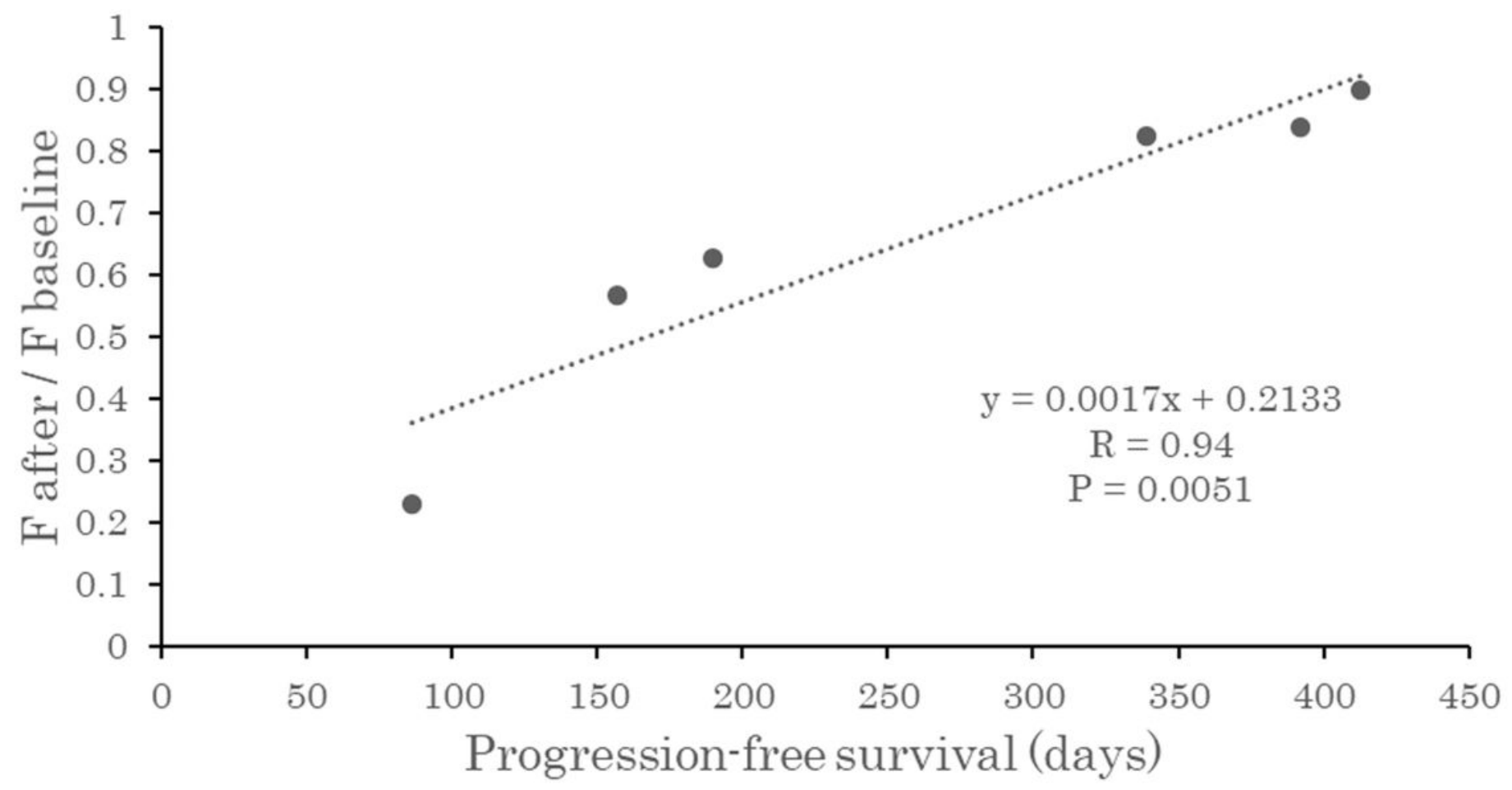

Figure 3

Relationships between change in tumor blood flow and progression-free survival in patients who underwent chemotherapy with bevacizumab The vertical line shows the ratio of tumor perfusion at the baseline to tumor perfusion after administration, and the horizon one shows progression-free survival. A substantial reduction in tumor perfusion after bevacizumab administration was associated with shorter time to progression. 


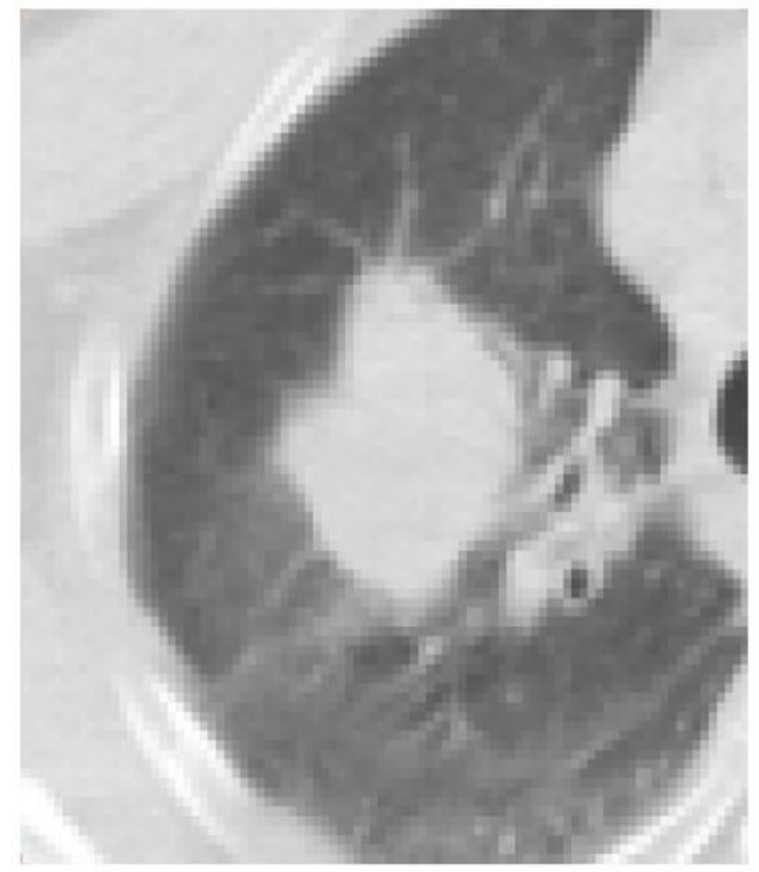

(a)

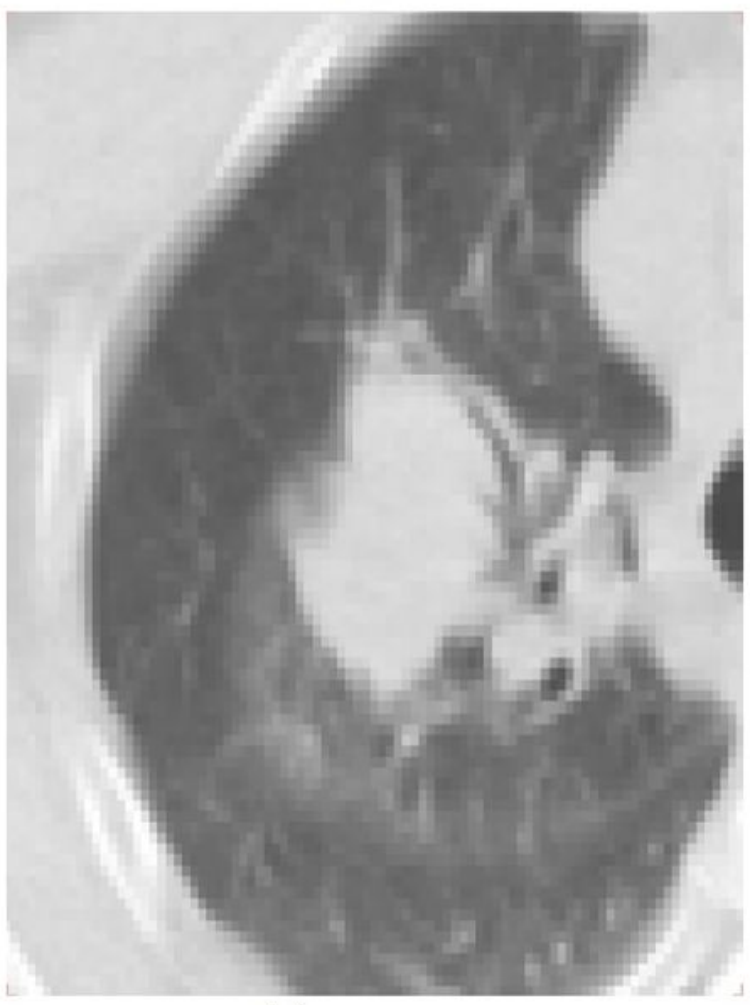

(c)

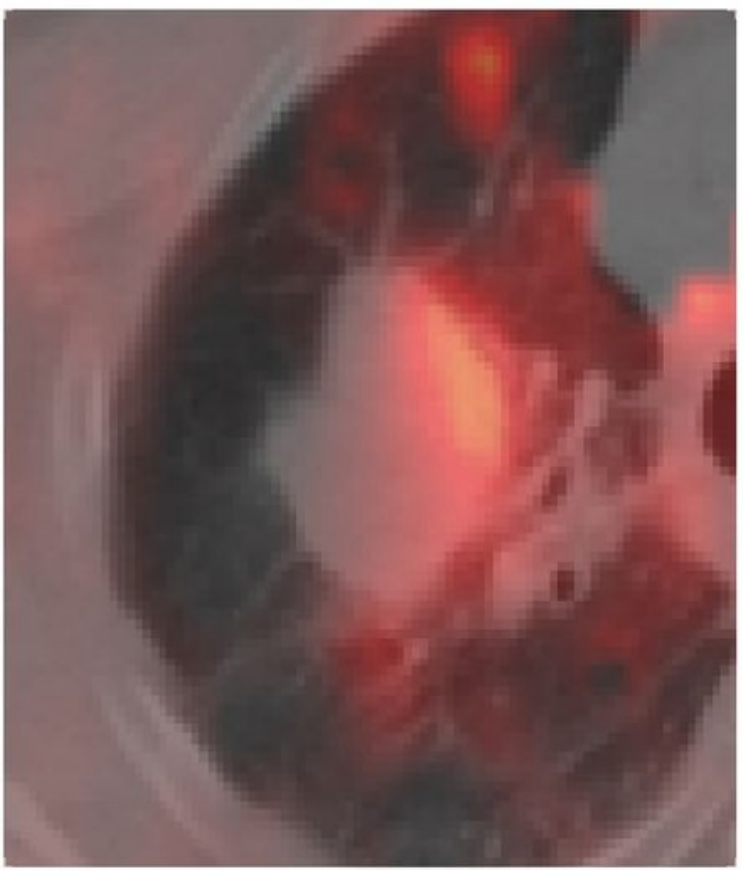

(b)

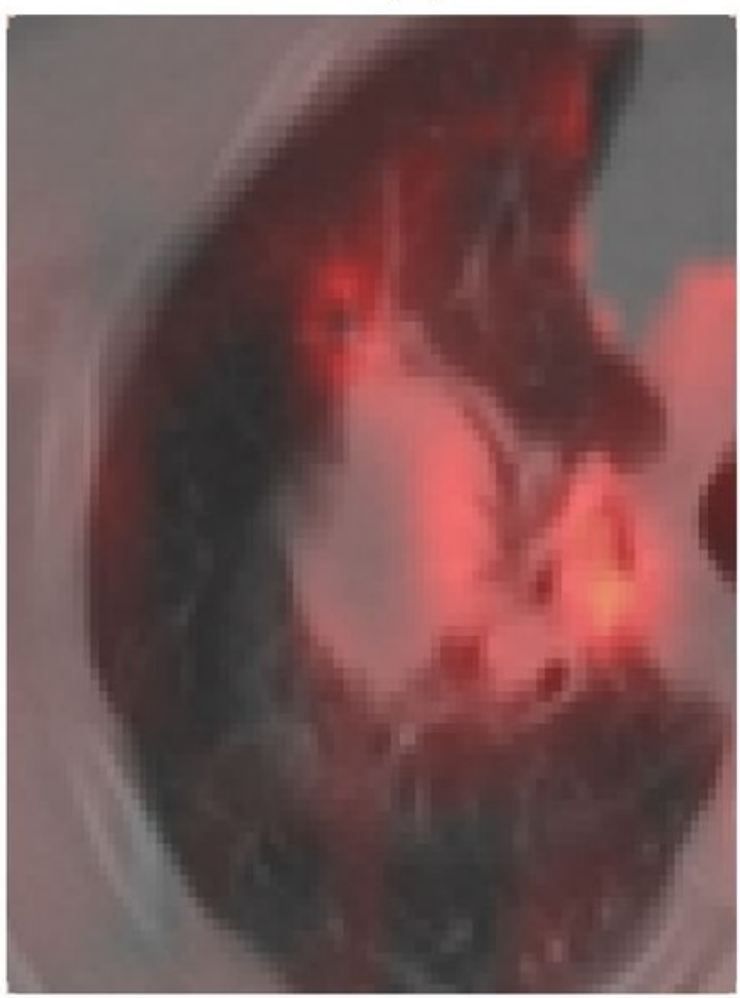

(d)

\section{Figure 4}

150-water PET image of 62-year-old man with adenocarcinoma treated with bevacizumab (patient no.1) $(a, b)$ CT and fusion image of CT and parametric image of blood flow at baseline. (c, d) CT and fusion image after chemotherapy. The CT showed a $4.4 \mathrm{~cm}$ large mass in the right lung. Tumor blood flow was $0.371 \mathrm{ml} / \mathrm{ml} / \mathrm{min}$ before treatment and slightly decreased to $0.334 \mathrm{ml} / \mathrm{ml} / \mathrm{min}$ after chemotherapy (blood flow ratio: 0.90). He had no tumor progression for 1 year and 2 months. 


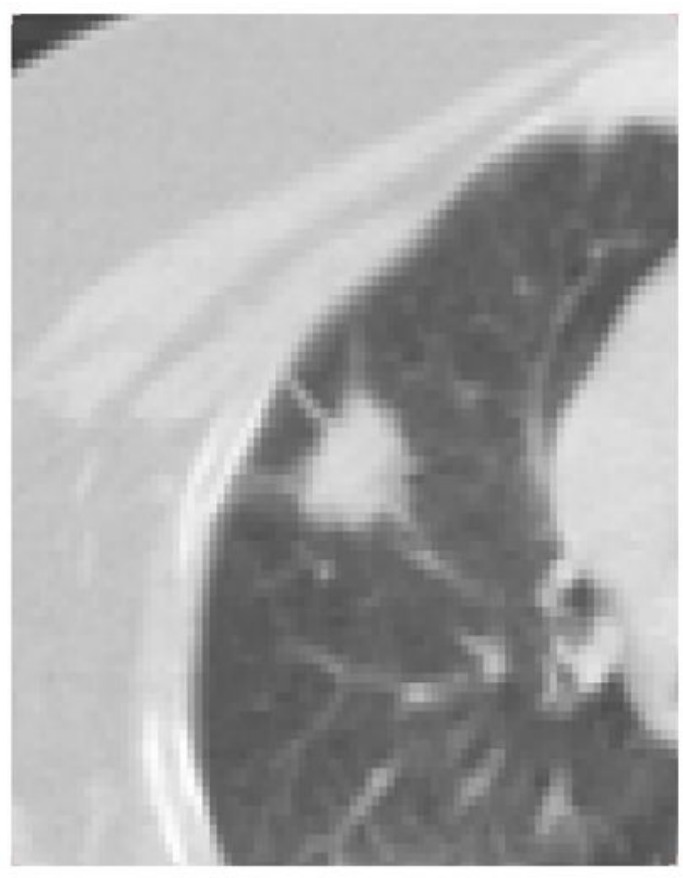

(a)

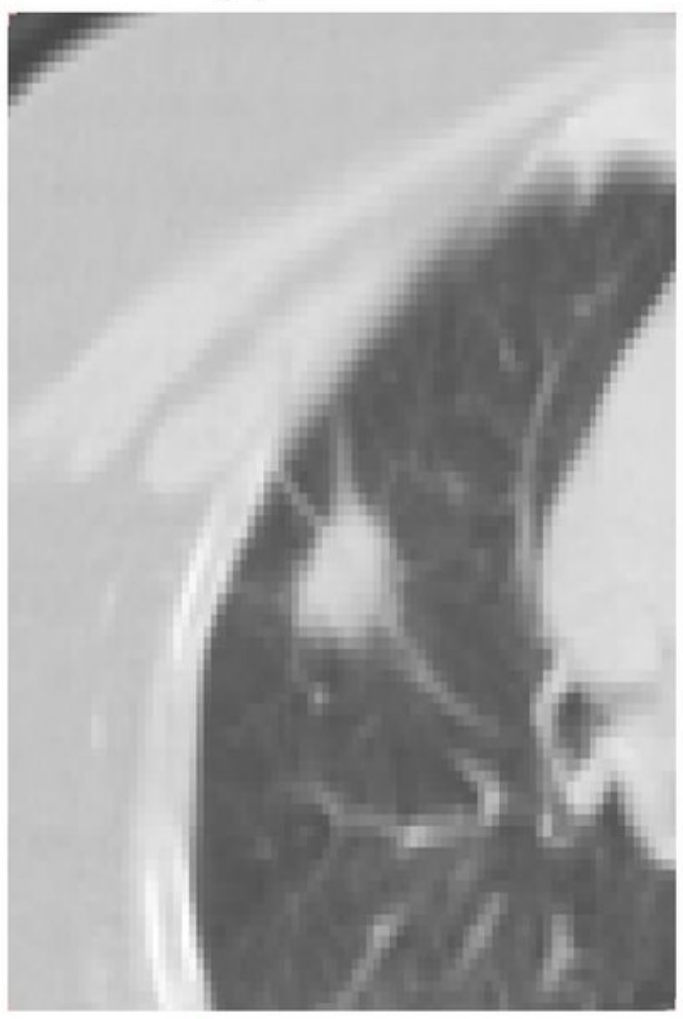

(c)

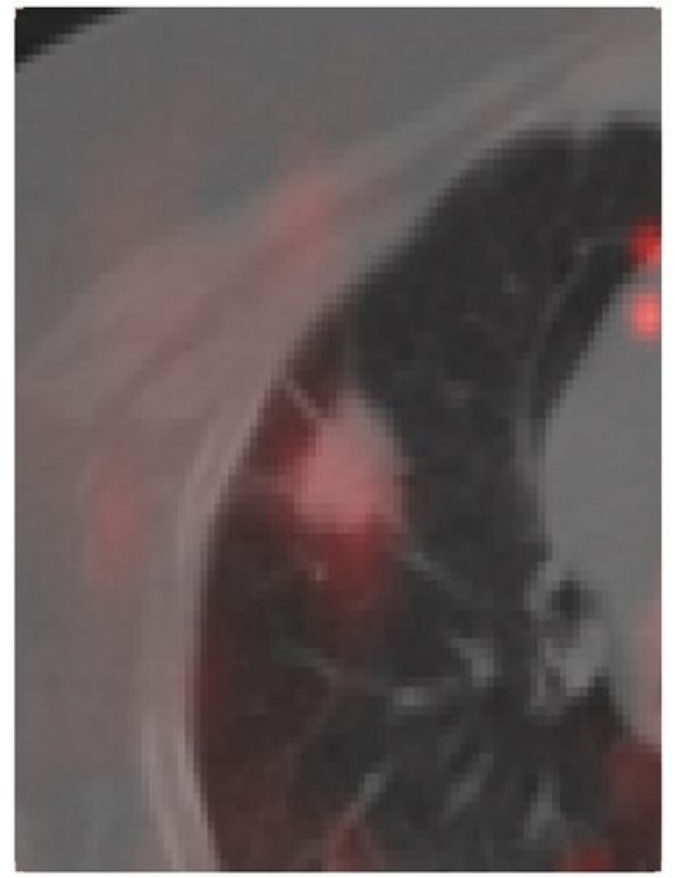

(b)

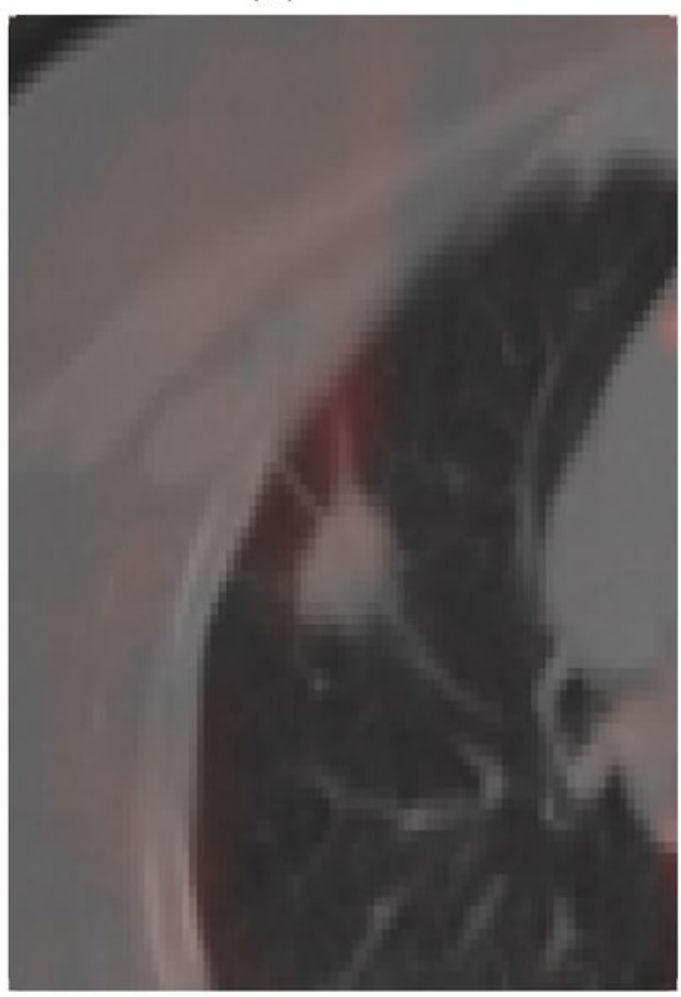

(d)

\section{Figure 5}

150-water positron emission tomography of 67-year-old woman with adenocarcinoma treated with bevacizumab (patient no.2) (a, b) CT and fusion image of CT and parametric image of blood flow at baseline. (c, d) CT and fusion image after chemotherapy. CT depicted a 2.6-cm nodule in the right lung. Tumor blood flow was markedly reduced, from $0.256 \mathrm{ml} / \mathrm{cm} 3 / \mathrm{min}$ to $0.059 \mathrm{ml} / \mathrm{cm} 3 / \mathrm{min}$ (blood flow ratio 0.23$)$. Three months later the tumor exhibited regrowth. 


\section{Supplementary Files}

This is a list of supplementary files associated with this preprint. Click to download.

- manuscriptsupplementary.pdf 